\title{
The Berkeley Spectrometer for ORFEUS: Laboratory and In-Flight Performance
}

\author{
MARK HURWITZ AND STUART BOWYER
}

\author{
Center for EUV Astrophysics, 2150 Kittredge Street, \\ University of California, Berkeley, CA $94720-5030$, USA
}

The Berkeley spectrometer aboard the ORFEUS payload achieved a variety of "firsts" during its inaugural mission in September 1993. The instrument utilizes spherical gratings with mechanically ruled varied line-spacing, and curved microchannel plate detectors with delay-line anode readout systems, to cover the $390-1200 \AA$ band at a resolution of $\lambda / 5000$. The instrument will be discussed, and its performance illustrated with calibration and in-flight spectra. Science highlights from the ORFEUS-I mission will be presented (oral presentation only). The payload will be available for use by guest investigators during the ORFEUS-II mission currently scheduled for late 1996.

\section{Introduction and Background}

ORFEUS, the Orbiting Retrievable Far and Extreme Ultraviolet Spectrometers, is a joint project of NASA and DARA, the German space agency. It is an outgrowth of a longstanding collaboration between Prof. Bowyer and the Space Astrophysics Group at UC Berkeley, and Prof. Grewing and the Astronomical Institute at the University of Tuebingen (AIT).

The ORFEUS telescope is 1 meter in diameter and 4 meters long (Grewing et al. 1991). The Berkeley spectrometer (Hurwitz \& Bowyer 1991) sits at the prime focus of the $f / 2.4$ normal incidence primary mirror. This instrument is designed to provide high-resolution $(\lambda / 5000)$ spectroscopy of point sources between 390 and $1200 \AA$, with an effective area of about $4-6 \mathrm{~cm}^{2}$. Alternatively, an off-axis paraboloidal mirror can be driven into the light path, collimating the beam and directing it into an Echelle spectrometer provided by AIT and the Landessternwarte Heidelberg (LSW). That instrument is designed to provide $\lambda / 10,000$ spectroscopy of point sources between 900 and $1250 \AA$ at somewhat lower sensitivity.

The resolution of the ORFEUS spectrometers significantly exceeds that of other instruments with comparable sensitivity at wavelengths of overlap. Below $760 \AA$, the spectrometers on the Extreme Ultraviolet Explorer offer somewhat lower sensitivity (but longer observing times), and a resolution of $\lambda / 300$. Between 800 and $1250 \AA$, the Hopkins Ultraviolet Telescope offers higher sensitivity, and comparable observing times, but again the resolution is limited to about $\lambda / 300$. The Hubble Space Telescope exceeds ORFEUS performance above about $1170 \AA$. A smaller telescope mounted parallel to the ORFEUS optical axis is IMAPS, (Jenkins et al. 1988) provided by the Princeton University Observatory. IMAPS offers extremely high resolution $\left(\lambda / 2 \times 10^{5}\right)$ in the far ultraviolet (FUV), but more limited sensitivity.

ORFEUS flew successfully aboard the German space platform ASTRO-SPAS, which was deployed from the shuttle Discovery in September of 1993 and recaptured some 5 days later. During this inaugural mission (hereafter ORFEUS-SPAS), the Berkeley spectrometer functioned well. More than 100 individual pointings were successfully carried out with the Berkeley spectrometer, including multiple observations of faint sources. This

601 
Spherical Varied Line-Space Grating

(4x) on Focusing Mechanisms

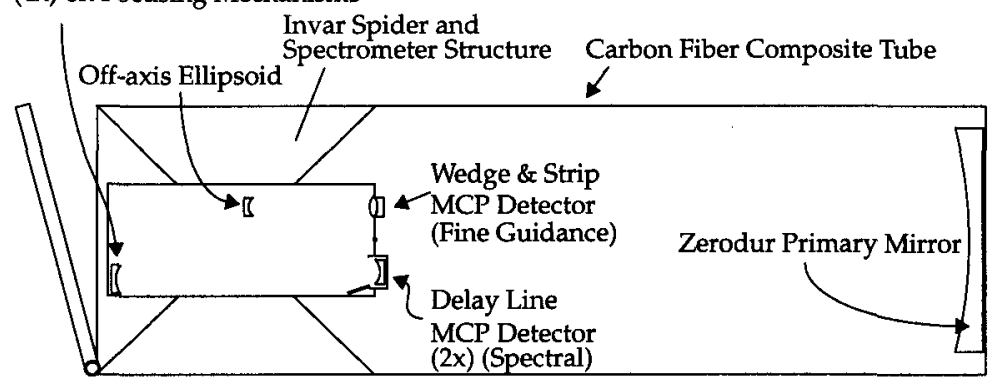

FIGURE 1. Cross-section schematic of ORFEUS telescope with Berkeley spectrometer.

total includes targets observed for the Berkeley science program, targets observed for our collaborators at AIT and LSW, and joint target observations.

The spacecraft has flown once since the ORFEUS-SPAS I mission, carrying a cryogenic infrared instrument for study of Earth's atmosphere (CRISTA-SPAS). A reflight of the ORFEUS instrument package is scheduled for late 1996. During this mission $50 \%$ of the available science time will be devoted to observations led by guest investigators, who may elect to utilize either of the ORFEUS spectrometers or IMAPS.

In this paper we discuss the performance of the Berkeley spectrometer in detail. The spacecraft and telescope are discussed insofar as they affect the spectra and the observing program.

\section{The Berkeley Spectrometer: General Principles and Design Details}

In Figure 1 we show the position of the Berkeley spectrometer within the ORFEUS telescope (the Echelle spectrometer is omitted for clarity). The Berkeley spectrometer occupies the inner $50 \%$ of the aperture diameter and obscures the inner $25 \%$ of the primary mirror area. For reference, the instrument $Z$ axis is parallel to the optical axis; the prime focus defines the plane of $Z=0$, and the $Z$ coordinate increases toward the top of the telescope.

Shadows from five spider vanes divide the monolithic telescope aperture into five segments of the outer annulus. This segmentation is illustrated in Figure 2, where we show an incoming "photon's view" of the system. Starlight from all five segments comes to a common focus some $240 \mathrm{~cm}$ from the primary mirror, then diverges into the Berkeley spectrometer volume.

As the beams from the five segments diverge, each segment of the annulus strikes a distinct optic within the Berkeley spectrometer. The smallest segment, representing a $36^{\circ}$ wedge of the annulus, is intercepted by an off-axis ellipsoidal optic near $Z=$ $+60 \mathrm{~cm}$ which images the target and aperture onto a sealed tube microchannel plate detector sensitive to wavelengths near $1500 \AA$. This fine guidance system is used for initial coalignment of the ORFEUS telescope and the external star tracker of the ASTRO-SPAS. It can also be used for postflight spectral reconstruction to correct for target "jitter" 


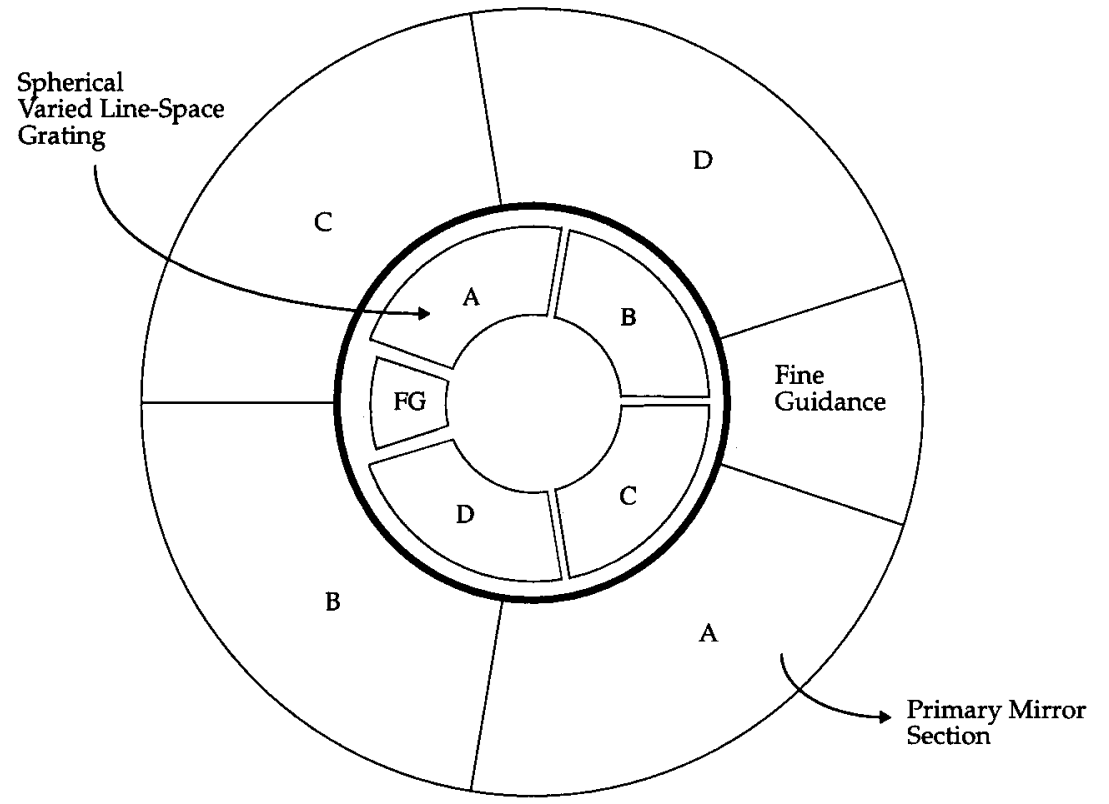

FIgURE 2. Telescope as "seen" by incoming photon. The $Z$ axis is perpendicular to page, pointing at viewer.

during an observation, although this was not necessary for spectra collected during the ORFEUS-SPAS I mission.

Each of the larger segments labeled A through D subtends $81^{\circ}$ of the annulus and strikes a distinct diffraction grating. The gratings are located at the extreme end of the spectrometer, near $Z=+100 \mathrm{~cm}$. The position of the gratings is shown in Figure 2; from this vantage point the viewer is seeing the "back side" of each grating, and of course, in the actual instrument the gratings are obscured by the surrounding spectrometer structure, thermal blankets, etc. Each grating disperses a unique sub-bandpass across one of two spectral detectors near the focal plane of the spectrometer. In Figure 3 we show the layout of detectors, including both the spectral and the fine guidance sealed tube, in the focal plane near $Z=0$. Note that the scale has been expanded by a factor of 2 in this figure.

The reasons for adopting this overall design are fairly straightforward and are discussed in Hurwitz \& Bowyer (1986). The telescope diameter and overall length are the maximum that can be accommodated within the spacecraft and shuttle envelope; a fast primary was a necessity. High-resolution spectroscopy required that either the aperture be subdivided into slower segments, or that a secondary mirror be introduced. The latter would have increased the effective focal length, imposing more severe restrictions on allowable spacecraft jitter. Furthermore, the potential gain in effective area (e.g., utilizing the entire beam vs. only a segment of it) would have been offset by the low reflectivity of normal incidence optics at EUV/FUV wavelengths. And as a practical matter the beam would have required subsequent subdivision in any case, given the very broad spectral bandpass (a factor of 3 in wavelength), the high spectral resolution that was desired, and the limited number of resolution elements provided by even the most advanced detector systems. 


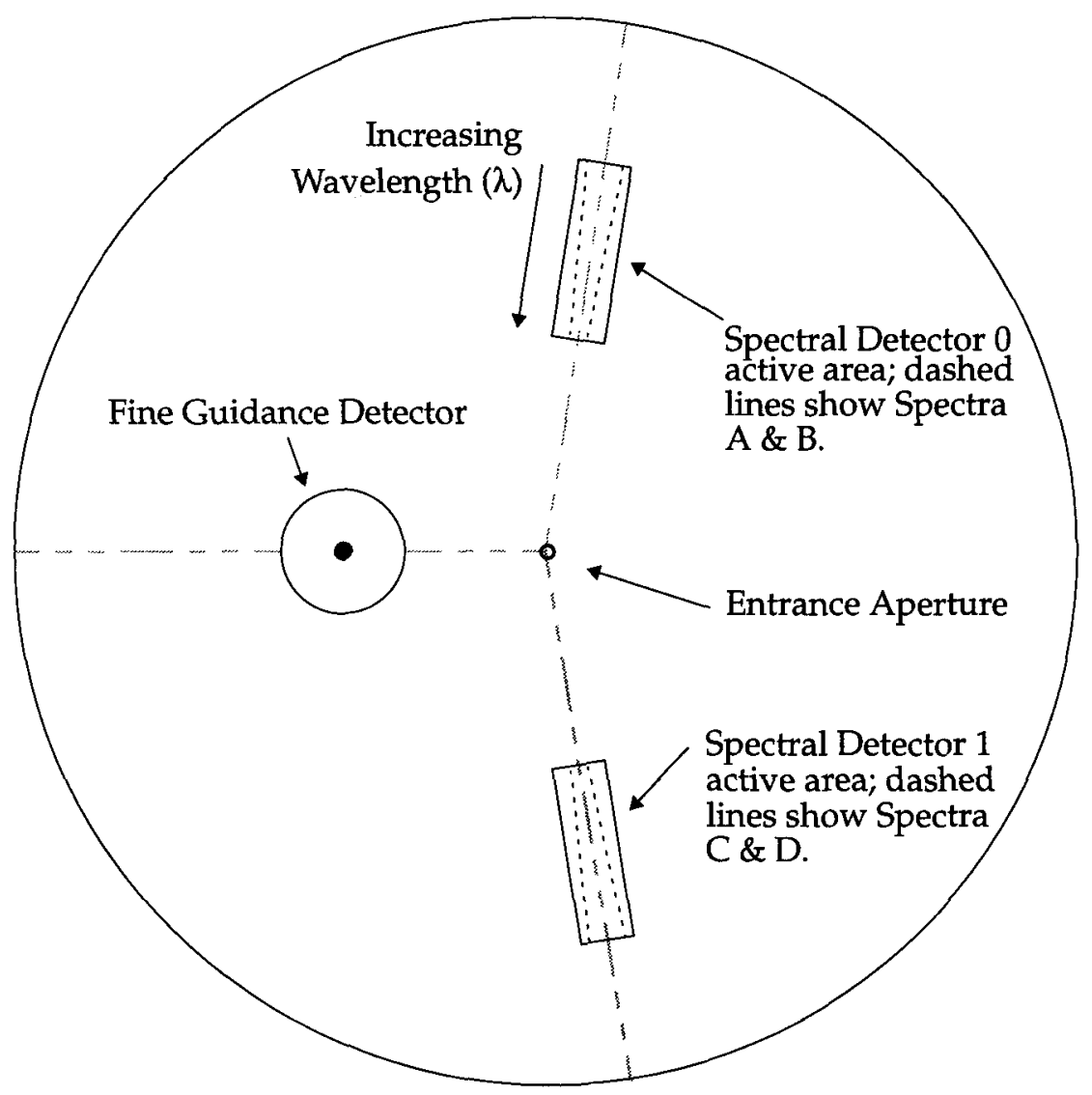

FIGURE 3. Focal plane detector layout.

TABLE 1.

Grating Central groove Nom. Bandpass

$\begin{array}{lllll} & \text { dens. }(/ \mathrm{mm}) & (\AA) & \text { (First Mission) } & \text { (Second Mission) } \\ \mathrm{A} & 6000.0 & 389-523 & \text { Evaporated Ir. } & \text { Evaporated Ir } \\ \mathrm{B} & 4550.0 & 513-690 & \text { Evaporated Ir } & \text { Evaporated Ir } \\ \mathrm{C} & 3450.4 & 676-910 & \text { Evaporated Ir } & \text { Sputtered SiC } \\ \mathrm{D} & 2616.6 & 892-1200 & \text { Evaporated Ir } & \text { Sputtered SiC }\end{array}$

The geometry of each grating/detector system is identical save for rotation about the $Z$ axis and/or reflection through the detector midplane. The incidence angle is $12^{\circ}$. Central groove densities, nominal bandpasses, and coatings are contained in Table 1 . The wavelength coverage $\left(\lambda_{\max } / \lambda_{\min }\right)$ of each grating is identical, as is the ratio of central wavelength to groove density.

When the design was first proposed, (e.g., Hurwitz \& Bowyer 1986) the optics were 
TABLE 2 .

$\begin{array}{clccc}\text { Dim. } & \text { Technique } & \text { Length }(\mathrm{mm}) & \text { Resolution }(\mu \mathrm{m}) & \text { Digitization }(\mu \mathrm{m}) \\ X & \text { Delay line } & 82 & 30-40 & 3.5 \\ Y & \text { Chg. division } & 26 & 200 & 120\end{array}$

toroidal in surface figure, with uniform line spacing, and followed the classical Rowland circle geometry. It was subsequently realized that higher resolution and reduction of astigmatism was possible using spherical optics with mechanically ruled varied linespacing (SVLS) in a non-Rowland mounting (Harada et al. 1991). Spectrometers of this type had been proposed earlier (Harada \& Kita 1980) but had never before been used in an astronomical application. The geometry enforced by the prime focus spectrograph position imposed unique constraints on the optical design. The adopted SVLS parameters, discussed in Harada et al. (1991), were optimized at Berkeley with an iterative raytracing technique.

The two spectral detectors incorporate curved microchannel plates with delay-line anodes (Siegmund et al. 1991) to encode the $X$ position of the dispersed photons. ORFEUSSPAS I was the first space flight for detectors of this type, and they performed extraordinarily well. Along the $Y$ axis, imaging is used only to separate the two spectra and to isolate the spectra from detector background; resolution requirements are modest. $Y$-axis imaging is provided by an interleaved wedge/wedge anode pattern and charge division. Spectral detector parameters are contained in Table 2. Both spectral detectors are housed in a sealed vacuum chamber equipped with doors; both utilize $\mathrm{KBr}$ photocathodes deposited on the front microchannel plate surface.

We now present relevant characteristics of other hardware systems before returning to system performance values.

\section{Spacecraft, Telescope, and Apertures}

The ASTRO-SPAS, fabricated by Daimler-Benz Aerospace (formerly Deutsche Aerospace), offers high scientific performance, but its resources are designed for short duration missions.

Absolute pointing is accurate to within a few arc seconds; compressed gas thrusters provide the necessary torques. An external star tracker provides a reference signal to the attitude/maneuvering control system (AMCS). During ORFEUS-SPAS I, the AMCS successfully placed almost every target within the $20^{\prime \prime}$ diameter aperture; one or two observations failed because of a lack of suitable guide stars.

Some tens of watts of power are provided to the experiments, using energy stored in batteries. Data are primarily archived by on-board tape recorders; about $130 \mathrm{kbits} \mathrm{s}^{-1}$ are allocated to the scientific experiments. This high speed (HS) channel records up to about 4000 spectral photon events per second, but cannot be accessed until the spacecraft is recovered and returned to Earth. A much slower (QL or quick-look) telemetry channel allows transmission of some $8 \mathrm{kbits} \mathrm{s}^{-1}$ of data to the ground in real time, but only during periods when the ASTRO-SPAS is in contact with the shuttle and the shuttle is in contact with the ground via TDRSS. The ASTRO-SPAS can record the data stream from only a single experiment at any given time. This limitation does not restrict functionality, since only one of the Berkeley or Echelle spectrometers can receive the ORFEUS telescope beam, and the targets that can be observed with IMAPS are too bright for either ORFEUS spectrometer. 
The ORFEUS telescope, fabricated by Kayser-Threde, contains three major mechanisms. One operates the large door at the $+Z$ extremum of the telescope. A second drives the collimator mirror used to direct the beam into the Echelle spectrometer. A third actuates an aperture blade at the prime focus. For ORFEUS-SPAS I, the science aperture was a circular hole $20^{\prime \prime}$ in diameter. In the second mission, observations with the Berkeley spectrometer will be carried out with three apertures near the prime focus. A $20^{\prime \prime}$ on-axis hole admits the light from the target and diffuse emission. A second $20^{\prime \prime}$ diameter hole is located off axis and admits diffuse emission only. These holes are displaced perpendicular to the dispersion direction, so their individual spectra will be separated on the spectral detectors, enabling diffuse emission to be subtracted from the target spectrum. A third hole, $60^{\prime \prime}$ in diameter is also located off axis, and is covered by a thin tin filter. In most applications the presence of this aperture can be ignored. The filter will be used only for extreme ultraviolet (EUV) observations of the bright B stars $\epsilon$ and $\beta \mathrm{CMa}$, whose integrated FUV flux could otherwise scatter from the gratings and overwhelm the EUV signal.

For a variety of reasons it was not practical to measure the end-to-end focus of the complete telescope and spectrometer system at EUV/FUV wavelengths prior to launch. The telescope and spectrometer were focused independently, and the overall system focus relied on mechanical mounting tolerances. The telescope tube is fabricated of carbonfiber composite, and is therefore prone to shrinkage as water vapor is outgassed on orbit. Thermal modeling of the Berkeley spectrometer indicated that our internal Invar structure would offer high dimensional stability, but the uncertainty in the thermal model was difficult to ascertain prior to flight experience. For all these reasons, we equipped the Berkeley spectrometer gratings with independent $Z$-axis focusing mechanisms, driven by stepper motors which can be actuated on orbit. (These mechanisms also facilitated preflight alignment and internal focusing in our laboratory calibration chamber.) The focusing period was scheduled early in the ORFEUS-SPAS I mission, but this period was occupied by critical but originally unscheduled checkout and alignment activities. Problems then arose in the telescope mechanisms; these too were eventually rectified, but for a time they threatened to end the science portion of the mission. When normal operations were restored, we put a premium on data gathering rather than fine tuning of the spectrometer performance. Evaluation of the real-time spectra verified that the onorbit focus was at least reasonably close to the laboratory value, so we did not attempt to reinsert a focusing activity in the remaining mission time line. Postflight analysis reveals some defocus; the in-flight resolution was $\lambda / 3000$.

\section{Performance: Resolution, Effective Area, and Backgrounds}

Various terms contribute to the net resolution error budget. Estimates for these terms are found in Table 3, both under ideal and realistic assumptions. Values are FWHM, in micrometers. The "ideal" column corresponds to a resolution of about $\lambda / 9500$. The "realistic" column corresponds to a resolution of about $\lambda / 5900$.

Preflight laboratory data confirm the realistic resolution to within about $10 \%$. Here jitter should be negligible, but the entrance pinhole is comparatively large $(25 \mu \mathrm{m})$. In these circumstances we expect a resolution of about $\lambda / 5500$. In Figure 4 we show a small section of a laboratory spectrum of argon emission lines near $544 \AA$ (Grating B). The two bright features in this figure are separated by $4.26 \AA$, and show FWHM values of almost precisely $\lambda / 5000$.

In Figure 5 we show a small section of the spectrum of a symbiotic binary star near the far-ultraviolet $O$ vi lines (Grating D) collected during the ORFEUS-SPAS I mis- 


\section{TABLE 3.}

\section{Term}

Primary mirror spot size

Grating aberrations (fab. and mounting)

Detector resolution

Defocus

Uncorrected jitter

TOTAL

$\begin{array}{cl}\text { Ideal } & \text { Realistic } \\ 8 & 12 \\ 4 & 15 \\ 30 & 40 \\ 0 & 20 \\ 0 & 12 \\ 31.3 & 50.1\end{array}$

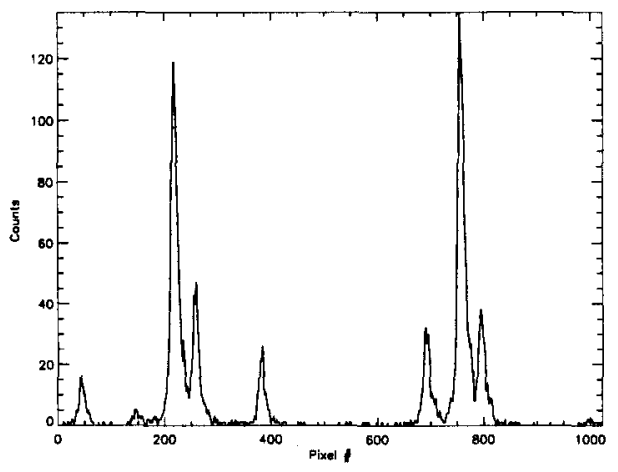

Figure 4. Section of a laboratory Grating B spectrum of argon.

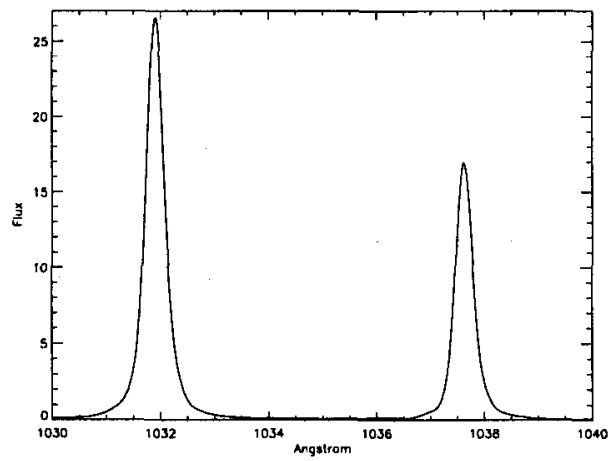

Figure 5. Section of a flight Grating D spectrum of a symbiotic binary star.

sion. The FWHM of these features is $\lambda / 3000$. Interstellar absorption lines in continuous spectra show this characteristic width, indicating that it is an instrumental limit, not an astrophysical effect. Spacecraft jitter, for which no corrections were applied, would limit the resolution at about $\lambda / 5400$. The most natural explanation for the $\lambda / 3000$ is a defocus somewhere in the system as discussed above.

The in-flight effective area can be estimated from observations of the well studied hot DA white dwarf G191-B2B (Vennes \& Fontaine 1992). In Figure 6 we show the in-flight effective area (solid line); this is somewhat below preflight estimates based on "theoretical" expectations for individual components, but not grossly so. The effective area was about $10 \%$ higher upon initial instrument turn-on, but quickly declined to the 


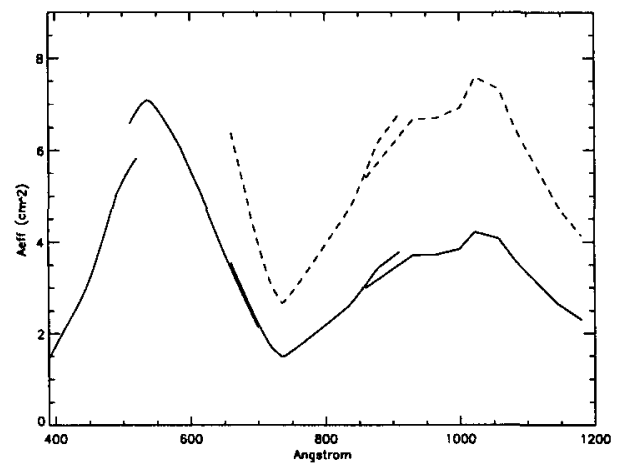

FIGURE 6. Spectrometer effective area during ORFEUS-SPAS I mission (solid), estimated -II mission (dotted).

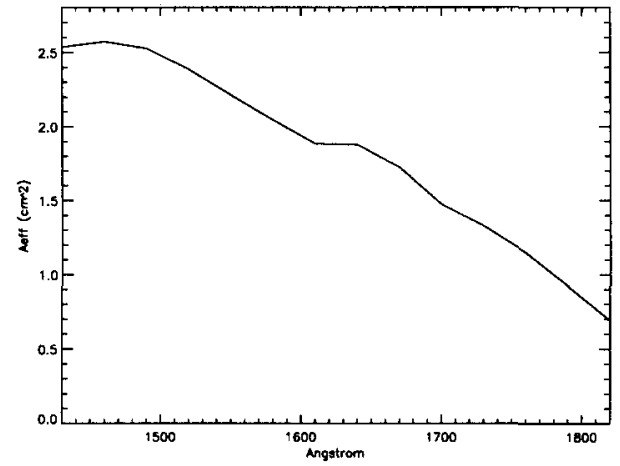

FIGURE 7. Fine Guidance effective area.

value shown here, then stabilized. This decline is presumably associated with an initial outgassing of contaminants, which then condensed on the optics and/or detector.

For the next mission, $\mathrm{SiC}$ coatings on Gratings $\mathrm{C}$ and $\mathrm{D}$ should improve the effective area, as shown by the dashed line in Figure 6 . The $10 \%$ on-orbit loss may have been recovered by postflight cleaning and recoating of the optics; however, it could easily recur soon after deployment. At the time of writing, the preflight calibration for the next mission has not been carried out.

The effective area of the fine guidance system, again estimated from ORFEUS-SPAS 1 observations of G191-B2B, is shown in Figure 7. The barium fluoride entrance window of the sealed tube reduces the effective area of this system to negligible values below about $1400 \AA$.

We acknowledge the support of NASA grant NAG5-696. The ORFEUS program is supported by DARA grant WE 350 OB 85013. 


\section{REFERENCES}

Grewing, M. ET AL. 1991, The ORFEUS Mission, in Extreme Ultraviolet Astronomy, ed. R. F. Malina \& S. Bowyer, New York: Pergamon, 437

Harada, T. \& Kita, T. 1980, Appl. Opt., 19, 3987

Harada, T., Kita, T., Hurwitz, M., \& BowYer, S. 1991, International Conference on the Application and Theory of Periodic Structures, Proc. SPIE, 1545, 2

HuRwitz, M. \& BowYer, S. 1986, A High Resolution Spectrometer for EUV/FUV Wavelengths, Proc. SPIE, 627, 375

HURwITZ, M. \& BowYER, S. 1991, The Berkeley EUV Spectrometer for the ORFEUS Mission, in Extreme Ultraviolet Astronomy, ed. R. F. Malina \& S. Bowyer, New York: Pergamon, 442

Jenkins, E. B., Joseph, C. L., Long, D., Zucchino, P. M. Carruthers, G. R. Bottema, M. \& Delamere, W. A. 1988, Proc. SPIE, 932, 213

Siegmund, O. H. W., Lampton, M., Raffanti, R., \& Herrick, W. 1991, High Resolution Delay Line Readouts for Microchannel Plates, Nuclear Instr. and Methods in Phys. Res., 310,311

Vennes, S. \& Fontaine, G. 1992, An Interpretation of the Spectral Properties of Hot Hydrogen-Rich White Dwarfs with Stratified H/He Model Atmospheres, ApJ, 401, 288 